\title{
The unsung reviewer
}

\author{
Manuscript peer reviewing is at the heart of the scientific system, but it seems that these duties are often not \\ properly (if at all) recognized by universities, funding agencies or even the rest of the scientific community.
}

S cientists wear many hats these days. They prepare and teach classes and sit on various committees. Then there are the multiple activities directly related to their research, including grant writing, mentoring students and postdocs, attending conferences, writing papers and reviewing manuscripts. All these duties can take a considerable amount of time and effort and most are recognized as worthy contributions by funding agencies, universities and research institutions when evaluating a scientist's performance. On the other hand, peer reviewing papers seems to be the Rodney Dangerfield ("I get no respect!") of a scientist's duties.

This situation has been highlighted by a recent report from the US Bipartisan Policy Center (http://www.bipartisanpolicy.org), a nonprofit organization whose "Science for Policy Project" (SPP) report aims to improve how science is used in the formulation of regulatory policies. Composed of a panel of 13 "ideologically diverse" experts from business, academia, government and nonprofit organizations, the SPP report contains four broad recommendations. The first three are for the US Administration and federal agencies, on the importance of distinguishing science questions from policy debates, how to form advisory panels and how to present the conclusions from literature reviews in an open and transparent way. The last recommendation is also directed at universities, scientific journals and scientists themselves, saying we all "can help improve the use of science in the regulatory process by strengthening peer review."

The SPP report notes that peer review is essential for the scientific system, but "scientists may feel too burdened to review their colleagues' papers or may do so with insufficient care. Peer review is no longer assumed to be a professional obligation." From our vantage point, we do not fully agree with the last statement. Our reviewer pool is very broad, and we find that scientists are usually very motivated to review for us. We also monitor the quality of the reviews we receive, and do not consult those reviewers who have given poor quality reviews in the past. We also know that our reviewers put a lot of effort into assessing the manuscripts and preparing thorough and constructive reports. We have conducted an informal survey among some of our regular reviewers, and found that reviewing each manuscript can take from 3 to 8 hours. They review around four manuscripts each month, sometimes fewer, depending on other duties. Across journals, they do decline many of the requests they receive.

In addition, the SPP report points to the need to increase the number of scientists who participate in peer review of draft manuscripts and makes specific suggestions for federal agencies, including listing such service on their grant applications or even making it a requirement for funding. In other words, grantees would need to peer review a minimum number of manuscripts during the time span of a grant to qualify for future funding. Universities should also do more to encourage their faculty members to participate in peer review, for instance, by considering such services as part of tenure, promotion and salary decisions. Some of our consulted reviewers agree that more credit should be given to peer reviewing activities, but others caution that it is hard to assess the quality of the work done and that junior faculty members should focus on their own research program, particularly in these financially constrained times.

Finally, the SPP report urges scientific journals to improve the quality of peer reviews, for example, by acknowledging top reviewers or giving reviewers feedback on the quality of their reviews, perhaps from the authors themselves. At Nature Structural \& Molecular Biology, we do provide our reviewers with editorial feedback, informing them of our decision and sending the comments from the other reviewers. We are sure most authors, in hindsight, would agree that reviewers' comments are helpful and constructive. This brings up a very important point: the primary purpose of the peer review process is to help the authors improve their paper, even if the work ultimately does not get published in NSMB. This might not be clear to less experienced reviewers, who may tend to focus on finding the flaws in a manuscript.

At $N S M B$, we do try to expand our pool of reviewers, often engaging younger scientists in the process, but experience makes a difference. In that regard, the SPP report notes that graduate students, postdocs and even junior faculty members should be mentored on peer review. Our reviewers told us they often engage junior members of their group in the review process. We find that practice healthy, as long as the official reviewer properly supervises the process. The reviewers do this not to delegate this important task, but because it is a valuable learning opportunity. In fact, co-reviewing a paper with students or postdocs does not reduce the time spent by the reviewer on each manuscript—quite the opposite. One reviewer told us about having two or three members of the lab read the manuscript and participate in a group discussion before writing the report. Although this makes the process substantially longer, everybody enjoys and learns from the experience.

Peer review is often compared to jury duty, a chore that one has to do once in a while as a service to the community. But reviewing manuscripts can be enjoyable and useful at any career stage. True, it takes time, but as one reviewer aptly put it: "I still learn do's and don'ts from reviewing papers, as well as just getting useful information, so it is time spent pretty well." It is also true that one does not get much open recognition from it, and there are limitations on what the journals can do without compromising the reviewers' anonymity. We have in the past publicly acknowledged our reviewers at the end of the year, and will do so again this year. The SPP report stresses that it is important to stress that "peer reviewing manuscripts should be an expected and appreciated aspect of a scientist's career." Of course, we do have a vested interest in this, but we firmly believe that it's important to cultivate a vibrant 'reviewer culture'. 